\title{
Short communication: Role of Streptococcus pluranimalium in Mediterranean buffaloes (Bubalus bubalis) with different udder health statuses
}

\author{
J. Guccione, ${ }^{\star 1}$ V. Perreten, $†$ A. Steiner, $\ddagger$ A. Thomann, $†$ A. Pesce,§ P. Ciaramella, ${ }^{*}$ and M. Bodmer \\ *Department of Veterinary Medicine and Animal Productions, University of Napoli "Federico II," Via Delpino 1, 80137 Napoli, Italy \\ †Institute of Veterinary Bacteriology, Department of Infectious Diseases and Pathobiology Vetsuisse-Faculty, and \\ ¥Clinic for Ruminants, Department of Clinical Veterinary Medicine, Vetsuisse-Faculty, University of Bern, Bern 3001, Switzerland \\ §Istituto Zooprofilattico del Mezzogiorno, Via A. Jervolino, 81100 Tuoro, Caserta District, Italy
}

\section{ABSTRACT}

The aims of the current study were to describe presence and clinical role over time of Streptococcus pluranimalium isolated in milk samples of Mediterranean buffalo (MB). Two hundred composite milk samples originating from 40 primiparous $\mathrm{MB}$ were collected at 10, 30, 60, 90, and $150 \mathrm{~d}$ in milk (DIM) and from 20 pluriparous MB at 77 to 120 DIM. Milk samples were used for analysis of somatic cell counts, bacteriological cultures, and identification (matrix-assisted laser desorption/ionization time-of-flight mass spectrometry). Nine of 200 (4.5\%) samples of primiparous MB and 3 of $20(15 \%)$ samples of pluriparous MB were positive for Strep. pluranimalium. The prevalence of the bacterium in primipari was $0 \%(0 / 40)$ at 10,30 , and 150 DIM, whereas it was $5(2 / 40)$ and $17.5 \%(7 / 40)$ at 60 and 90 DIM, respectively. Eight primipari were positive only once, whereas 1 was positive at 2 different samplings. Mono-infection was not detected in any of the age categories or udder health status. Infections were transient in primipari. Clinical mastitis was observed in primipari once at 90 DIM, subclinical mastitis detected twice in the same animals at 60 and 90 DIM, and intramammary infections were diagnosed 1 and 5 times at 60 and 90 DIM in primipari, respectively, whereas 3 infections were diagnosed in pluripari. The clinical reflections demonstrate for the first time the presence of Strep. pluranimalium in MB and its association with different udder health status. Nevertheless, it cannot be excluded that the bacterium may simply follow a pattern of commensal or opportunistic behavior, taking advantage of a preexisting bacterial udder infection.

Key words: Streptococcus pluranimalium, Mediterranean buffalo, mastitis, matrix-assisted laser desorption/ionization time-of-flight mass spectrometry

Received August 20, 2015.

Accepted December 6, 2015.

${ }^{1}$ Corresponding author: jacopo.guccione@unina.it

\section{Short Communication}

Streptococcus pluranimalium was described for the first time by Devriese et al. (1999a) in dairy cows. Since then, it has been isolated from several species of domestic animals and identified as cause of septicemia and valvular endocarditis in broiler chickens (Hedegaard et al., 2009) and as cause of mastitis, meningoventriculitis, abortion, stillbirth, vulvitis, vaginitis, and metritis in cows (Devriese et al., 1999b; Seimiya et al., 2007; Foster et al., 2010). Streptococcus pluranimalium infection has been also recently reported in humans, suggesting a zoonotic potential of this bacterium (Paolucci et al., 2013; Ayasinghe et al., 2014). Although Strep. pluranimalium has been isolated from milk samples of dairy cows with IMI in Belgium, its role as a mastitis pathogen in ruminants has not been clarified yet (Devriese et al., 1999b); it belongs to the large group of esculin-hydrolyzing, catalase-negative, gram-positive cocci (PNC), and its presence in mastitic milk may have been overlooked or misidentified with Streptococcus acidominimus or other $\alpha$-hemolytic streptococci using only basic phenotypic identification methods (Devriese et al., 1999b; Ulbegi-Mohyla et al., 2009). The PNC isolated from clinical and subclinical mastitis cases in dairy cows are commonly categorized as Streptococcus uberis in routine diagnostic laboratories (Devriese et al., 1999b). At present, the evaluation of the biochemical properties represents one of the most widespread used techniques to consistently distinguish Strep. pluranimalium from other PNC (Twomey et al., 2012). With the introduction of matrix-assisted laser desorption/ionization time-of-flight mass spectrometry (MALDI-TOF MS) technology in veterinary diagnostics, bacteria, including Streptococcus spp., can now be accurately identified to the species level, revealing a broader spectrum of possible mastitis pathogens (Schabauer et al., 2014). Although Mediterranean buffaloes (MB) and cows are frequently recognized as similar hosts for udder pathogens causing mastitis (Guccione et al., 2014a,b), Strep . pluranimalium has not been re- 
ported as a cause of udder disorders in MB. Thus, the aims of the current study were to (1) describe for the first time the presence of Strep. pluranimalium in milk samples of MB, (2) describe the respective clinical outcomes, elucidating the potential pathway of infection over time, and (3) draw preliminary conclusions on the clinical relevance.

Forty primiparous MB (from 10 to 150 DIM) and 20 pluriparous MB (ranging from second to fifth lactation and from 77 to 120 DIM), originating from a breeding farm of 600 dairy buffaloes and located in southern Italy (Caserta district), were enrolled in the present study. All animals were chosen by convenience sampling between January and June 2013. Before parturition until 40 to 50 DIM, the primipari were kept separated from the pluripari. Milking was performed in the same parlor, but the primipari were housed separately and always milked first. After 50 DIM, the primipari and pluripari were mixed, including the milking procedure. Milk samples were aseptically collected in sterile test tubes (BD Vacutainer, Oxford, UK) immediately before regular evening milking, as described for dairy cows by the National Mastitis Council (2004). At 10, 30, 60, 90, and 150 DIM, 2 composite milk samples (40 mL each) were created by mixing equal amounts of milk from all 4 quarters. The first was used to determine the SCC and bacteriological culture following the procedure described in a previous study (Guccione et al., 2014a,b); the second was immediately frozen $\left(-20^{\circ} \mathrm{C}\right)$ until further investigations with culture and species identification by MALDI-TOF MS analysis. At the time the sampling of primipari was completed (150 DIM), 1 composite milk sample of $10 \mathrm{~mL}$ each was collected once aseptically from 20 pluriparous $\mathrm{MB}$ of the same herd and frozen at $-20^{\circ} \mathrm{C}$ for subsequent bacteriological analysis (culture and MALDI-TOF MS). If identification at genus level was not possible, and in case of growth of mixed flora, the bacteriological identification was performed at species level as described by Frey et al. (2013). Plates showing bacterial growth from 1 up to 3 different colonies were subcultured on tryptic soy agar with sheep blood (TSA-SB) and further identified using MALDI-TOF MS (Microflex LT; Bruker Daltonics GmbH, Billerica, MA). Cultures morphologically containing more than 3 different bacteria were considered as mixed flora (contamination). In this case, only colonies displaying characteristics for streptococci where further purified and identified using MALDI-TOF MS.

All the putative Strep. pluranimalium strains isolated from buffalo milk generated similar profiles and were identified as Strep. pluranimalium with scores situated between 1.9 and 2.1. A profile of Strep. pluranimalium strain M2141 has been included into the MALDI-TOF database as a reference and all the strains have been reanalyzed, all generating profiles matching to M2141 with scores above 2.1 to confirm similarity. Sequence analysis of the 16S rRNA gene of Strep. pluranimalium strain M2141 (GenBank accession no. KT943470) from buffalo milk revealed a $99.5 \%$ identity to that of the type strain Strep. pluranimalium T70 (GenBank accession no. NR_104971), and only 95.6 and $96.5 \%$ to those of the next closest related Streptococcus species, Streptococcus thoraltensis type strain S69 (GenBank accession no. NR_026368) and Streptococcus hyovaginalis type strain SHV515 (GenBank accession no. NR_044912), respectively; this indicated that the buffalo strain belonged to the Strep. pluranimalium species group (Twomey et al., 2012).

On the days of sampling, each MB enrolled was individually submitted to a complete clinical examination, with particular focus on udder health status. Local and systemic signs and changes in milk characteristics were also recorded during each sampling. California Mastitis Test (CMT) was routinely performed from each composite milk sample, with values $\geq 1$ interpreted as positive (Guccione et al., 2014b). Electrical conductivity (EC) and daily milk yield (DMY) were recorded using automatic dedicated software (Afifarm, Afimilk, Kibbutz Afikim, Israel). Composite milk samples were placed in a cooled box $\left(4^{\circ} \mathrm{C}\right)$ and brought to the reference laboratory within $1 \mathrm{~h}$ of collection, when SCC analyses were performed by means of flow cytometry (Fossomatic 5000, Foss Electric, Hillerød, Denmark). Buffaloes' udder health status was classified as healthy, affected by IMI, subclinical mastitis (SCM) and clinical mastitis (CM; considering the results of the udder's clinical examination), SCC, and bacteriological culture as previously described by Guccione et al. (2014a,b). Different animal health status, SCC, and DMY were analyzed by standard descriptive statistics, and data distribution was assessed using histograms, normal probability plots, and Shapiro Wilk tests. Data were expressed as absolute numbers, percentage, or mean \pm SD; SCC were expressed as $\log _{10}($ cells $/ \mathrm{mL})$. Untransformed and log-transformed variables were described using box plots and compared using parametric (Student's $t$-test) and nonparametric test ( $\chi^{2}$-test). Probabilities of $<0.05$ were considered significant. Data were analyzed using dedicated software (SPSS, Version 17.0, SPSS Inc., Chicago, IL).

Of all the milk samples collected during this study, 9 of $200(4.5 \%)$ samples of primipari and 3 of $20(15 \%)$ samples of pluripari were positive for Strep. pluranimalium. The prevalence of the bacterium in primipari was $0 \%(0 / 40)$ at 10,30 , and 150 DIM, whereas it was $5(2 / 40)$, and $17.5 \%(7 / 40)$ at 60 and 90 DIM, respectively. In total, 8 of 9 primipari were positive to 
Strep. pluranimalium only one time, whereas 1 of 9 was positive at 2 consecutive samplings (60 and 90 DIM). Mono-infection with Strep. pluranimalium was not detected in any age category of MB. In primpari, co-infection of Strep. pluranimalium was associated once with Staphylococcus haemolyticus and once with Staphylococcus rostri at 60 DIM and 6 times with Staphylococcus aureus and once with Staph. rostri at 90 DIM (Table 1). In pluriparous MB $(\mathrm{n}=20)$, Strep. pluranimalium was found twice associated with Staph. aureus and once with Staph. dysgalactiae. In 5 cases, Strep. pluranimalium was detected within a mixed flora of more than 3 other bacteria types (Table 1). During the current study, 9 of $200 \mathrm{CM}, 11$ of 200 SCM, and 136 of 200 IMI were recorded within the sampling population of primiparous MB; of these, CM due to co-infection between Strep. pluranimalium and other udder pathogens was observed once at 90 DIM (Strep. pluranimalium + Staph. aureus), SCM was detected twice in the same primiparous MB at 60 (Strep. pluranimalium + Staph. haemolyticus) and 90 DIM (Strep. pluranimalium + Staph. aureus), whereas IMI was diagnosed in primipari 1 (Strep. pluranimalium + Staph. rostri) and 5 times [Strep. pluranimalium + Staph. haemolyticus (4), Strep. pluranimalium + Staph. rostri (1)] at 60 and 90 DIM, respectively. Sixty-three out of 200 primipari were considered healthy at least in 1 sampling. Regarding the pluriparous MB, 15 of 20 animals were affected by IMI (5/20 samples were contaminated); of the 15 pluripari with IMI, co-infections between Strep. pluranimalium and other udder bacteria was recorded 3 times [Staph. aureus (2); Staph. dysgalactiae (1)].

During the current study, CMT was always positive in samples of primipari with CM $(\mathrm{n}=1)$ and SCM $(\mathrm{n}=2)$ due to co-infection of the udder with Strep. pluranimalium; it was negative in 5 and positive in 1 sample of primipari with IMI, whereas it was negative in $84.1 \%(53 / 63)$ and positive in $15.9 \%(10 / 63)$ samples of primiparous MB classified as healthy. A statistically significant difference was found concerning CMT values observed in healthy and positive primiparous MB $(P$ $<0.05)$. Somatic cell count values ranged from 3.20 to $5.90 \log _{10}$ cells $/ \mathrm{mL}$ (mean $=4.47 \pm 0.55$ cells $/ \mathrm{mL}$ ) in healthy primipari and from 4.20 to $6.06 \log _{10}$ cells/ $\mathrm{mL}$ (mean $=4.79 \pm 0.58$ cells $/ \mathrm{mL}$ ) in those primipari positive for Strep. pluranimalium $(P<0.02$, Figure 1$)$. The EC values ranged from 6.90 to $10.20 \mathrm{mS} / \mathrm{cm}$ (mean $=8.24 \pm 0.51 \mathrm{mS} / \mathrm{cm}$ ) in healthy primipari and from 7.80 to $12.2 \mathrm{mS} / \mathrm{cm}$ (mean $=8.62 \pm 1.37 \mathrm{mS} / \mathrm{cm})$ in those primipari positive for Strep pluranimalium $(P=$ $0.21)$; the DMY ranged from 5.0 to $18.0 \mathrm{~kg} / \mathrm{d}(10.79 \pm$ $2.53 \mathrm{~kg} / \mathrm{d}$ ) in healthy primipari and from 7.6 to 14.2 $\mathrm{kg} / \mathrm{d}(11.18 \pm 2.37 \mathrm{~kg} / \mathrm{d})$ in those primipari positive to Strep. pluranimalium $(P=0.32)$. Regarding the pluriparous $\mathrm{MB}$, the CMT was always positive in the animals co-infected with Strep. pluranimalium $(3 / 3)$, the $\mathrm{SCC}$ was $5.16 \pm 0.15 \log _{10}$ cells $/ \mathrm{mL}$, the EC was 8.40 $\pm 0.40 \mathrm{mS} / \mathrm{cm}$, and the DMY was $12.29 \pm 0.64 \mathrm{~kg} / \mathrm{d}$. A statistically significant difference was observed concerning SCC values between healthy primiparous MB and pluriparous MB positive for Strep. pluranimalium $(P<0.01$, Figure 1$)$, but none regarding EC, CMT, and DMY.

Our study describes, for the first time, the presence of Strep. pluranimalium in milk samples of MB. This was made possible by the use of MALDI-TOF MS as an identification method that results in a rapid and reliable modern technique to identify veterinary bacteria (Randall et al., 2015). As this technology is still not frequently established in veterinary diagnostic laboratories, it is not always possible to estimate the prevalence of these bacteria in mastitis milk of both dairy cattle and buffaloes. However, Strep. pluranimalium has already been recognized as an udder pathogen in cases of SCM of dairy cows (Devriese et al., 1999b), and its role as mastitis pathogen in buffaloes deserves closer

Table 1. Number and type of bacteria isolated from 4 quarter composite milk samples with Streptococcus pluranimalium co-infections of 40 primiparous buffaloes at 10,30,60,90, and 150 DIM and 20 pluriparous buffaloes at 77 to 120 DIM (udder health status is given in parentheses)

\begin{tabular}{|c|c|c|c|c|c|}
\hline \multirow[b]{2}{*}{ Item } & \multicolumn{5}{|c|}{ DIM at time of sampling ${ }^{1}$} \\
\hline & 10 & 30 & 60 & 90 & 150 \\
\hline Strep. pluranimalium, Staphylococcus aureus & - & - & - & $\begin{array}{c}6 \\
{[\operatorname{IMI}(4), \operatorname{SCM}(1), \mathrm{CM}(1)]}\end{array}$ & - \\
\hline Strep. pluranimalium, Staphylococcus rostri & - & - & 1 (IMI) & 1 (IMI) & - \\
\hline Strep. pluranimalium, Staphylococcus haemolyticus & - & - & $1(\mathrm{SCM})$ & - & - \\
\hline Strep. pluranimalium, Streptococcus dysgalactiae & - & - & - & - & 1 (IMI) \\
\hline Strep. pluranimalium, Staph. haemolyticus & - & - & - & - & 1 (IMI) \\
\hline Strep. pluranimalium, mixed flora & - & - & - & - & 5 (contaminated) \\
\hline
\end{tabular}

${ }^{1} \mathrm{SCM}=$ subclinical mastitis; $\mathrm{CM}=$ clinical mastitis. 
attention. Our study revealed that colonization of the udder of MB with Strep. pluranimalium only occurs transiently, as a co-infection with udder pathogens, and may be associated with various degrees of clinic signs. In primiparous MB, Strep. pluranimalium was not detected in samples taken at 10 and 30 DIM but was noted for the first time at 60 DIM. This phenomenon may have been evident, as primipari were kept separate from and always milked before the adult buffaloes during this period, and mixing of the 2 groups occurred after 40 and 50 DIM. After the appearance of Strep. pluranimalium in primiparous $\mathrm{MB}$, a supplementary milk sample collection was performed once from the pluriparous MB sharing the same paddock to evaluate the hypothesis of a reservoir role of the udder of pluripari for Strep. pluranimalium.

Additional investigations in pluriparous MB did not reveal any mono-infection due to Strep. pluranimalium (15\% of co-infections with udder pathogens and $25 \%$ of contaminated samples). Furthermore, a difference of IMI prevalence due to Strep. pluranimalium between primiparous and pluriparous MB was not found. These findings support an alternative hypothesis that the environment of the pluripari represents the reservoir, whereas the teat skin and teat cup liner may of the pluripari represent the inanimate vectors of the bacteria. Clear evidence supporting the role of the bacteria

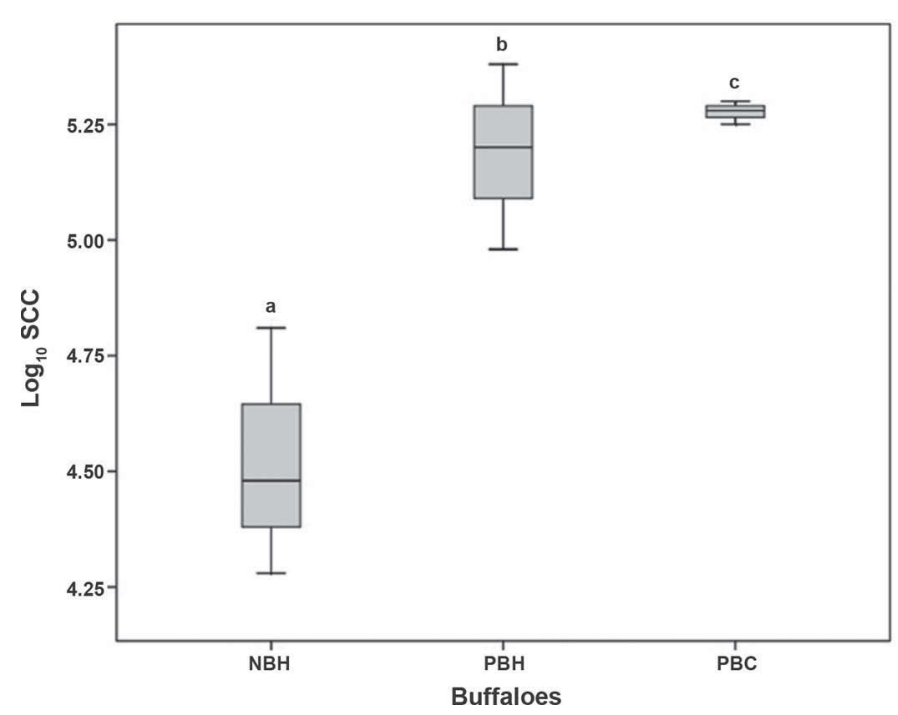

Figure 1. Box plot of $\log _{10}$ SCC distributions of milk samples collected from Mediterranean buffaloes. NBH = buffalo heifer milk samples negative for Streptococcus pluranimalium; $\mathrm{PBH}$ and $\mathrm{PBC}=$ buffalo heifer and buffalo cow milk samples positive for Strep. pluranimalium, respectively. Statistically significant differences were found between SCC values of the groups NBH and PBH $(\mathrm{a}, \mathrm{b}=P<0.02)$ and of the groups NBH and PBC $(\mathrm{a}, \mathrm{c}=P<0.01)$. The box plot shows the mean of $\log _{10}$ SCC distributions of milk samples collected from Mediterranean buffaloes. as a specific udder pathogen of MB was not found. The clinical outcomes associated with the colonization of the udders with $S$. pluranimalium have not yet been described for buffaloes or cattle. Although some clinical parameters, such CMT, EC, and DMY, seem to not be significantly associated with the presence of Strep. pluranimalium, our study shows a significant difference of mean SCC values between healthy primipari and primipari positive for Strep. pluranimalium (CM, SCM, and IMI). Because mono-infection of the udder with Strep. pluranimalium was not observed, it seems possible that the clinical signs at the udder level were mainly caused by the other pathogens identified (Staph. aureus, Staph. haemolyticus, and Staph. rostri). Staphylococcus aureus, Staph. haemolyticus, and Staph. rostri are widely acknowledged as associated with increased SCC values when detected in an intramammary mono-infection (Locatelli et al., 2013; Guccione et al., 2014a,b). It cannot be excluded that Strep. pluranimalium simply followed a pattern of commensal or opportunistic behavior, taking advantage of a preexisting bacterial udder infection and causing clinical disease only, if at all, if favorable conditions are given. The udder is not likely to represent the main target organ of this bacterium, which is supported by the fact that the infection was transient in all primipari.

As indicated by several studies in human medicine, the zoonotic potential of Strep. pluranimalium is still uncertain and requires further investigations (Paolucci et al., 2013; Ayasinghe et al., 2014). Considering the results of the present study, we cannot exclude a potential risk for human health related to the consumption of raw buffalo milk. Thus, the role of Strep. pluranimalium in the dairy food chain warrants investigations in more detail. Additionally, as Strep. pluranimalium appeared to be present within buffalo herds and considering its association with cases of abortion in dairy cows (Foster et al., 2008), the role of this opportunistic pathogen as a causative agents of abortion and metritis in buffaloes needs to be closely monitored.

In conclusion, the clinical reflections of the present investigation demonstrated for the first time the presence of Strep. pluranimalium in a herd of MB and its association with different udder health status. Our study clearly showed that the udder of some primiparous MB became rapidly colonized with Strep. pluranimalium when they shared the same environment as infected pluripari. Although the bacteria was isolated from milk samples of animals with CM, SCM, and IMI, the absence of mono-infections, the inconsistent increase of the SCC, and the transient colonization of the udder in primipari suggest that Strep. pluranimalium may not represent an udder-specific pathogen in MB. Nevertheless, the role of Strep. pluranimalium in the etiology 
of other pathologies in MB and its zoonotic potential warrants further investigations.

\section{REFERENCES}

Ayasinghe, L., S. Sabbar, Y. Kazim, L. M. Awan, and H. K. N. Khan. 2014. Streptococcus pluranimalium: A novel human pathogen? Int. J. Surg. Case Rep. 5:1242-1246.

Devriese, L. A., J. Hommez, H. Laevens, B. Pot, P. Vandamme, and F. Haesebrouck. 1999b. Identification of aesculin-hydrolyzing streptococci, lactococci, aerococci and enterococci from subclinical intramammary infections in dairy cow. Vet. Microbiol. 70:87-94.

Devriese, L. A., P. Vandamme, M. D. Collins, N. Alvarez, B. Pot, J. Hommez, P. Butaye, and F. Haesebrouck. 1999a. Streptococcus pluranimalium sp. nov.: From cattle and other animals. Int. J. Syst. Bacteriol. 49:1221-1226.

Foster, G., J. Barley, F. Howie, E. Falsen, E. Moore, D. F. Twomey, P. Wragg, A. M. Whatmore, and E. Stubberfield. 2008. Streptococcus pluranimalium in bovine reproductive disease. Vet. Rec. 163:638.

Foster, G., L. Hunter, G. Baird, M. S. Koylass, and A. M. Whatmore. 2010. Streptococcus pluranimalium in ovine reproductive material. Vet. Rec. 166:246.

Frey, Y., J. P. Rodriguez, A. Thomann, S. Schwendener, and V. Perreten. 2013. Genetic characterization of antimicrobial resistance in coagulase-negative staphylococci from bovine mastitis milk. J. Dairy Sci. 96:2247-2257.

Guccione, J., A. Cosandey, A. Pesce, A. Di Loria, M. Pascale, D. Piantedosi, A. Steiner, H. U. Graber, and P. Ciaramella. 2014b. Clinical outcomes and molecular genotyping of Staphylococcus aureus isolated from milk samples of dairy primiparous Mediterranean buffaloes (Bubalus bubalis). J. Dairy Sci. 97:7606-7613.

Guccione, J., A. Pesce, M. Pascale, N. Tommasini, F. Garofalo, A. Di Loria, L. Cortese, C. Salzano, and P. Ciaramella. 2014a. Short communication: Effects of systemic treatment with penethamate hydriodide on udder health and milk yields in dry primiparous Mediterranean buffaloes (Bubalus bubalis). J. Dairy Sci. 97:22192225 .

Hedegaard, L., H. Christensen, M. S. Chadfield, J. P. Christensen, and M. Bisgaard. 2009. Association of Streptococcus pluranima- lium with valvular endocarditis and septicaemia in adult broiler parents. Avian Pathol. 38:155-160.

Locatelli, C., S. Piepers, S. De Vliegher, A. Barberio, K. Supré, L. Scaccabarozzi, G. Pisoni, V. Bronzo, F. Haesebrouck, and P. Moroni. 2013. Effect on quarter milk somatic cell count and antimicrobial susceptibility of Staphylococcus rostri causing intramammary infection in dairy water buffaloes. J. Dairy Sci. 96:3799-3805.

National Mastitis Council. 2004. Procedures for Collecting Milk Samples in Microbiological Procedures for the Diagnosis of Bovine Udder Infection and Determination of Milk Quality. National Mastitis Council Inc., Madison, WI.

Paolucci, M., M. Stanzani, F. Melchionda, G. Tolomelli, G. Castellani, M. P. Landini, S. Varani, R. E. Lewis, and V. Sambri. 2013. Routine use of a real-time polymerase chain reaction method for detection of bloodstream infections in neutropaenic patients. Diagn. Microbiol. Infect. Dis. 75:130-134.

Randall, L. P., F. Lemma, M. Koylass, J. Rogers, R. D. Ayling, D. Worth, M. Klita, A. Steventon, K. Line, P. Wragg, J. Muchowski, M. Kostrzewa, and M. A. Whatmore. 2015. Evaluation of MALDI$\mathrm{ToF}$ as a method for the identification of bacteria in the veterinary diagnostic laboratory. Res. Vet. Sci. 101:42-49.

Schabauer, L., M. Wenning, I. Huber, and M. Ehling-Schulz. 2014 Novel physico-chemical diagnostic tools for high throughput identification of bovine mastitis associated gram-positive, catalasenegative cocci. BMC Vet. Res. 10:156.

Seimiya, Y. M., M. Takahashi, T. Kudo, and K. Sasaki. 2007. Meningoventriculitis caused by Streptococcus pluranimalium in a neonatal calf of premature birth. J. Vet. Med. Sci. 69:657-660.

Twomey, D. F., T. Carson, G. Foster, M. S. Koylass, and A. M. Whatmore. 2012. Phenotypic characterisation and 16S rRNA sequence analysis of veterinary isolates of Streptococcus pluranimalium. Vet. J. 192:236-238.

Ulbegi-Mohyla, H., A. A. Hassan, T. Kanbar, J. Alber, C. Lämmler, E. P. Berninghoff, R. Weiß, U. Siebert, and M. Zschöck. 2009. Synergistic and antagonistic hemolytic activities of bacteria of genus Arcanobacterium and CAMP-like hemolysis of Arcanobacterium phocae and Arcanobacterium haemolyticum with Psychrobacter phenylpyruvicus. Res. Vet. Sci. 87:186-188. 